\title{
Joint optimization of source and relay precoding for AF MIMO relay systems
}

\author{
Jun $\mathrm{Li}^{1}$, Xueqin Jiang ${ }^{2}$, Sangseob Song ${ }^{1}$, Ying Guo ${ }^{3}$ and Moon Ho Lee ${ }^{1 *}$
}

\begin{abstract}
In this paper, we investigate a joint source and relay precoding design scheme for an amplify-and-forward (AF) multiple-input multiple-output (MIMO) relay system with absence of the direct link. The joint optimization problem, which is to minimize an objective function based on the mean square error (MSE), is formulated as a nonconvex optimization problem in the AF MIMO relay system. Instead of the conventional iterative method, we use an inequality to derive a lower bound of the MSE under the power constraint for obtaining a suboptimal solution of the objective function, which makes the optimization problem convex and also approaches the existing upper bound of the MSE, especially at the high signal-to-noise ratio (SNR). Numerical results show that this scheme outperforms the previous schemes in terms of either MSE or bit error rate (BER).
\end{abstract}

Keywords: Amplify-and-forward (AF) multiple-input multiple-output (MIMO) relay; Joint precoding; Lower bound

\section{Introduction}

As the relay channel was initially introduced in wireless networks $[1,2]$, the cooperative relay communication has been developed rapidly these days [3]. The known relay protocols have been classified as amplify-andforward (AF), decode-and-forward (DF), and compressand-forward (CF) [4].

Compared with DF and CF protocols, the AF protocol suffers from the noise enhancement, but it is still considered as a hot issue in wireless networks since it usually leads to low complexity and low consumption of power. On the other hand, the multiple-input multipleoutput (MIMO) technology was introduced to increase the channel capacity and improve the reliability of wireless networks in [5]. Therefore, using the MIMO technology into a relay system and the optimization design in the MIMO relay system have gained much attention [6].

The main optimizing processing of an AF MIMO relay system is to maximize or minimize objective functions, such as mutual information (MI), mean square error (MSE), sum of rate and signal-to-interference-plusnoise ratio (SINR). For example, Fang et al. proposed an approach to maximize the MI for an optimal design of

\footnotetext{
*Correspondence: moonho@jbnu.ac.kr

1 Department of Electronics and Information Engineering, Chonbuk National University, Baekje Road, Jeonju, South Korea

Full list of author information is available at the end of the article
}

source covariance matrix and relay matrix [7]. Similar results were achieved while taking a source covariance matrix as an identity matrix $[8,9]$. In addition, an optimization of the joint power constraint was designated to maximize the MI [10]. The minimization of the MSE for MIMO relay systems was derived for a joint optimal design of source matrix and relay precoding matrix [11]. Furthermore, unified frameworks were developed to optimize the source and relay precoding matrix while designing an iterative algorithm to allocate the optimal power to the relay channels [12]. Due to the high computational complexity of the iterative algorithm, a suboptimal algorithm was also developed to reduce its computational complexity $[13,14]$. As for the precoding multi-relay networks, the joint source-relay optimization design was proposed to maximize SINR [15]. The optimization of achievable rate and channel capacity was also derived [16]. Moreover, the optimizations of two-way relay systems were investigated using the precoding approach in a similar scenario as the previous literatures [17-19]. For the optimization of the AF MIMO relay systems, Sanguinetti et. al. not only summarized various kinds of optimization problems but also suggested several related solutions for each problem [20].

In this paper, we suggest a joint optimal design of the source and relay precoding matrices for AF MIMO relay

\section{Springer}

(C) $2015 \mathrm{Li}$ et al. Open Access This article is distributed under the terms of the Creative Commons Attribution 4.0 International License (http://creativecommons.org/licenses/by/4.0), which permits unrestricted use, distribution, and reproduction in any medium, provided you give appropriate credit to the original author(s) and the source, provide a link to the Creative Commons license, and indicate if changes were made. The Creative Commons Public Domain Dedication waiver (http://creativecommons. org/publicdomain/zero/1.0/) applies to the data made available in this article, unless otherwise stated. 
systems. For simplicity, we assume that the perfect channel state information (CSI) is available at the relay and destination. We will derive an objective function on the basis of the MSE. Since the proposed objective function is not convex, we further derive a lower bound of the objective function to make it convex which is different from the upper bound in [14]. The numerical results show that the lower bound has a better performance than the previous schemes. It approaches to the known upper bound at the high signal-to-noise ratio (SNR).

The rest of this paper is organized as follows. In the "System model" section, we introduce the system model for the AF MIMO relay system. The lower bound of the MSE is derived in the "Lower bound of MSE" section. In the "Numerical results" section, numerical results are presented. The "Conclusions" section concludes this paper.

Notations: Boldface upper- and lowercase letters denote matrices and column vectors, respectively. $(\cdot)^{H}$ stands for Hermitian transpose. $\mathbb{C}$ represents the complex number field. $\mathbf{I}_{M}$ is an identity matrix of size $M \times M \cdot \mathcal{C N}(\mu, v)$ stands for the complex Gaussian distribution with mean $\mu$ and covariance $v . E\{\cdot\}$ denotes the expectation operator. $\operatorname{tr}\{\cdot\}$ and $\operatorname{rank}\{\cdot\}$ denote the trace and rank of a matrix. $\mathbf{A}_{i j}$ denotes the $(i, j)$-th element of matrix A. $(\cdot)^{-1}$ stands for matrix inversion. $\nabla^{2}(\cdot)$ denotes the second-order gradient of a function. $(\cdot) \succeq 0$ stands for a semi-positive definite matrix.

\section{System model}

We consider an AF MIMO relay system as shown in Fig. 1, where the source, the AF relay and the destination are equipped with $N_{s}, N_{r}$ and $N_{d}$ antennas, respectively. The half-duplex mode is used for this system, where each node cannot transmit and receive simultaneously. The direct link is not considered and the flat fading is applied for all channels.

The transmission will take two time slots. In the first time slot, the source transmits a symbol vector $\mathbf{s} \in \mathbb{C}^{K}$ to the relay, where $E\left\{\mathbf{s s}^{H}\right\}=\mathbf{I}_{K}$. The received signal at the relay can be described as

$$
\mathbf{y}_{r}=\mathbf{H}_{1} \mathbf{W}_{1} \mathbf{s}+\mathbf{n}_{1},
$$

where $\mathbf{H}_{1} \in \mathbb{C}^{N_{r} \times N_{s}}$ denotes the channel matrix between the source and the relay, $\mathbf{W}_{1} \in \mathbb{C}^{N_{s} \times K}$ denotes the source precoding matrix and $\mathbf{n}_{1}$ denotes a Gaussian noise vector with $\mathbf{n}_{1} \sim \mathcal{C N}\left(0, \delta_{1}^{2} \mathbf{I}_{N_{r}}\right)$. For the simplicity, the power constraint $P_{1}$ at the source is given by

$$
\operatorname{tr}\left\{\mathbf{W}_{\mathbf{1}} \mathbf{W}_{\mathbf{1}}{ }^{H}\right\} \leq P_{1} .
$$

In the second time slot, the relay forwards the received signal after using a precoding matrix $\mathbf{W}_{2} \in \mathbb{C}^{N_{r} \times N_{r}}$. With the power constraint $P_{2}$ at the relay, we can obtain

$$
\operatorname{tr}\left\{\mathbf{W}_{2}\left(\mathbf{H}_{1} \mathbf{W}_{1} \mathbf{W}_{1}^{H} \mathbf{H}_{1}^{H}+\delta_{1}^{2} \mathbf{I}_{\mathbf{N}_{\mathbf{r}}}\right) \mathbf{W}_{2}^{H}\right\} \leq P_{2} .
$$

Subsequently, the received signal at the destination can be derived as

$$
\mathbf{y}_{\mathbf{d}}=\mathbf{H}_{2} \mathbf{W}_{2} \mathbf{H}_{1} \mathbf{W}_{1} \mathbf{s}+\mathbf{H}_{2} \mathbf{W}_{2} \mathbf{n}_{1}+\mathbf{n}_{2},
$$

where $\mathbf{H}_{2} \in \mathbb{C}^{N_{d} \times N_{r}}$ denotes the channel matrix between the relay and the destination and $\mathbf{n}_{2}$ denotes a Gaussian noise vector with $\mathbf{n}_{2} \sim \mathcal{C N}\left(0, \delta_{2}^{2} \mathbf{I}_{N_{d}}\right)$. In the end, a linear receiver $\mathbf{G} \in \mathbb{C}^{k \times N_{d}}$ is applied at the destination. Therefore, the estimated signal at the destination can be achieved as

$$
\overline{\mathbf{s}}=\mathbf{G y}_{d} .
$$

\section{Lower bound of MSE}

In order to derive an optimization processing with a lower bound for the AF MIMO relay system, we consider the MSE matrix given by

$$
\begin{aligned}
& \mathbf{M}\left(\mathbf{W}_{1}, \mathbf{W}_{2}\right)=E\left\{(\overline{\mathbf{s}}-\mathbf{s})(\overline{\mathbf{s}}-\mathbf{s})^{H}\right\} \\
& \quad=E\left\{\mathbf{G y}_{\mathbf{d}} \mathbf{y}_{\mathbf{d}}{ }^{H} \mathbf{G}^{H}-\mathbf{G y}_{\mathbf{d}} \mathbf{s}^{H}-\mathbf{s y}_{\mathbf{d}}{ }^{H} \mathbf{G}^{H}\right\}+\mathbf{I} .
\end{aligned}
$$

Substituting (4) and (5) into (6), we obtain

$$
\mathbf{M}\left(\mathbf{W}_{1}, \mathbf{W}_{2}\right)=\mathbf{G R}_{\mathbf{y}_{\mathbf{d}}} \mathbf{G}^{H}-\mathbf{G H}-\mathbf{H}^{H} \mathbf{G}^{H}+\mathbf{I},
$$

where the whole channel matrix $\mathbf{H}$, the noise covariance matrix $\mathbf{R}$ and the covariance matrix of the received signal $\mathbf{R}_{\mathbf{y}_{\mathbf{d}}}$ are described as follows

$$
\begin{aligned}
& \mathbf{H}=\mathbf{H}_{2} \mathbf{W}_{2} \mathbf{H}_{1} \mathbf{W}_{1}, \\
& \mathbf{R}=\delta_{1}^{2} \mathbf{H}_{2} \mathbf{W}_{2} \mathbf{W}_{2}^{H} \mathbf{H}_{2}^{H}+\delta_{2}^{2} \mathbf{I}_{N_{d}}, \\
& \mathbf{R}_{\mathbf{y}_{\mathbf{d}}}=\mathbf{H H}^{H}+\mathbf{R} .
\end{aligned}
$$

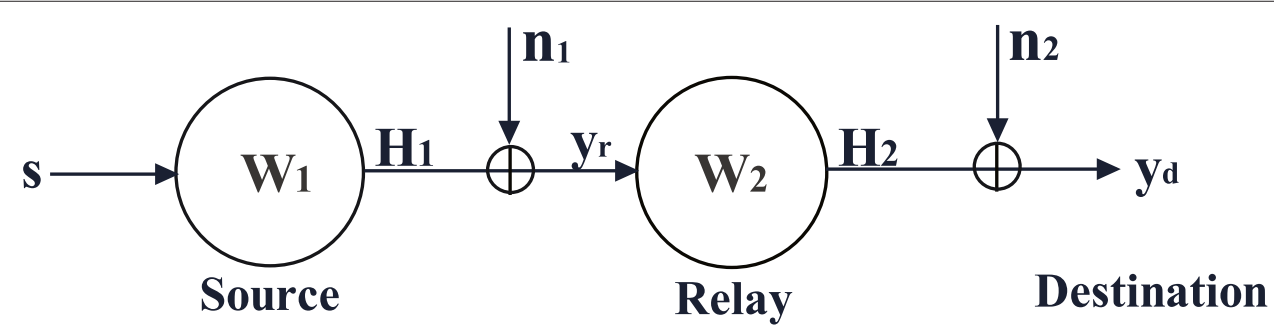

Fig. 1 The system model for the AF MIMO relay system 


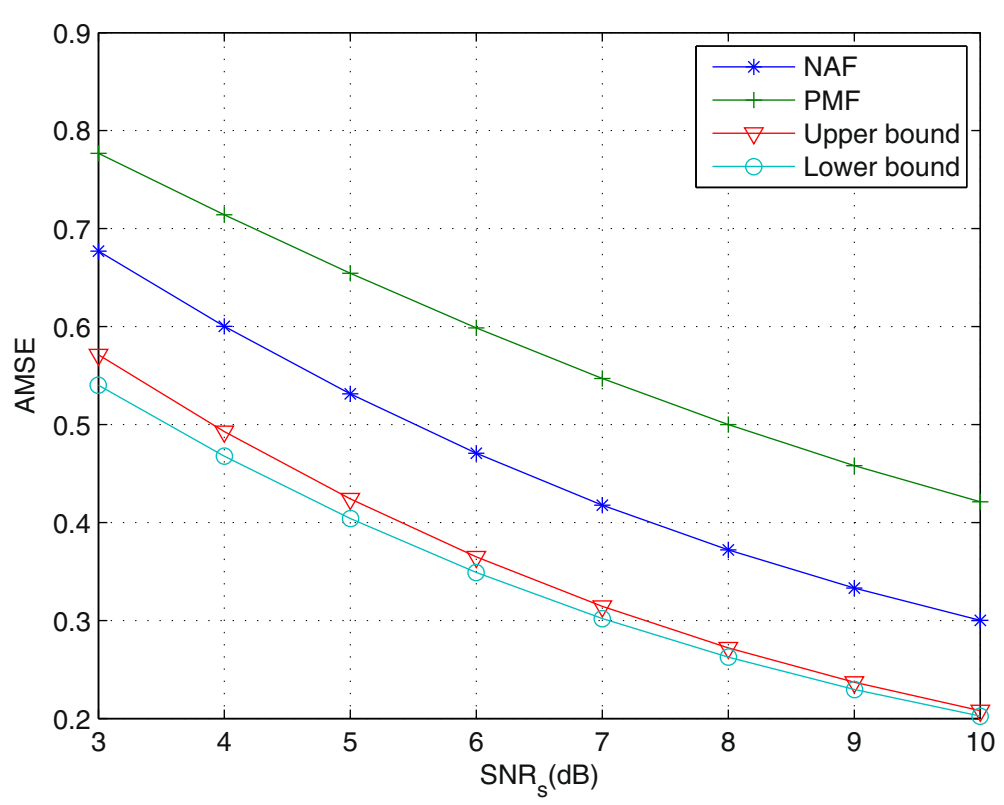

Fig. 2 AMSE vs SNR $. N_{s}=N_{r}=N_{d}=3$

The matrix $\mathbf{G}$ to minimize the MSE matrix is given by Wiener filter, i.e.,

$$
\mathbf{G}=(\mathbf{H})^{H}\left(\mathbf{H} \mathbf{H}^{H}+\mathbf{R}\right)^{-1} .
$$

By substituting (11) into (7), the minimal MSE matrix can be derived as

$$
\begin{aligned}
\mathbf{M}\left(\mathbf{W}_{1}, \mathbf{W}_{2}\right) & =\mathbf{I}-\mathbf{H}^{H}\left(\mathbf{H} \mathbf{H}^{H}+\mathbf{R}\right)^{-1} \mathbf{H} \\
& =\left(\mathbf{H}^{H} \mathbf{R}^{-1} \mathbf{H}+\mathbf{I}_{K}\right)^{-1},
\end{aligned}
$$

which is achieved on a basis of the matrix inversion transformation

$$
(\mathbf{A}+\mathbf{B C D})^{-1}=\mathbf{A}^{-1}-\mathbf{A}^{-1} \mathbf{B}\left(\mathbf{D A}^{-1} \mathbf{B}+\mathbf{C}^{-1}\right)^{-1} \mathbf{D A}^{-1} .
$$

In what follows, we will consider how to minimize the MSE matrix for the AF MIMO relay system. The arithmetic MSE (AMSE) [12] is given by

$$
\operatorname{AMSE}=\sum_{i=1}^{K}\left[\mathbf{M}\left(\mathbf{W}_{1}, \mathbf{W}_{2}\right)\right]_{i, i},
$$

where the MSE matrix $\mathbf{M}$ is chosen as a diagonal matrix. Then the SINR [21] can be expressed as

$$
\operatorname{SINR}=\sum_{i=1}^{K}\left(\frac{1}{\left[\mathbf{M}\left(\mathbf{W}_{1}, \mathbf{W}_{2}\right)\right]_{i, i}}-1\right) .
$$

It implies that minimizing the MSE is equivalent to maximizing the SINR. Also, the symbol error rate [22] can be described as

$$
P_{e}(\mathrm{SINR})=\alpha Q(\sqrt{\beta \mathrm{SINR}})
$$

where $\alpha$ and $\beta$ are constants that depend on the signal constellation, and $Q$ is the $Q$-function defined as $Q(x)=$ $(1 / \sqrt{2 \pi}) \int_{x}^{\infty} e^{-\lambda^{2} / 2} d \lambda$. Namely, minimizing the symbol error rate or bit error rate is also equivalent to minimizing the MSE. Using the abovementioned analysis, the optimal processing can be derived as

$$
\begin{aligned}
\min _{\mathbf{W}_{1}, \mathbf{W}_{2}} & {\left[\mathbf{M}\left(\mathbf{W}_{1}, \mathbf{W}_{2}\right)\right]_{i, i}, \quad 1 \leq i \leq K, } \\
\text { s.t. } & \operatorname{tr}\left\{\mathbf{W}_{\mathbf{1}} \mathbf{W}_{\mathbf{1}}{ }^{H}\right\} \leq P_{1}, \\
& \operatorname{tr}\left\{\mathbf{W}_{2}\left(\mathbf{H}_{1} \mathbf{W}_{1} \mathbf{W}_{1}^{H} \mathbf{H}_{1}^{H}+\delta_{1}^{2} \mathbf{I}_{\mathbf{N}_{\mathbf{r}}}\right) \mathbf{W}_{2}^{H}\right\} \leq P_{2} .
\end{aligned}
$$

Let us denote the singular value decomposition (SVD) of channels $H_{1}$ and $H_{2}$ as

$$
\mathbf{H}_{1}=\mathbf{U}_{1} \Lambda_{1} \mathbf{V}_{1}^{H},
$$

and

$$
\mathbf{H}_{2}=\mathbf{U}_{2} \Lambda_{2} \mathbf{V}_{2}^{H},
$$

where $\mathbf{U}_{1}, \mathbf{V}_{1}, \mathbf{U}_{2}$ and $\mathbf{V}_{2}$ are unitary matrices, while $\Lambda_{1}$ and $\Lambda_{2}$ are the diagonal matrices with entries being arranged in the non-increasing order [10]. In order to make the MSE matrix as a diagonal matrix, the optimal matrices $\mathbf{W}_{1}$ and $\mathbf{W}_{2}$ should be chosen as [12]

$$
\mathbf{W}_{1}=\overline{\mathbf{V}}_{1} \Sigma_{1},
$$

and

$$
\mathbf{W}_{2}=\overline{\mathbf{V}}_{2} \Sigma_{2} \overline{\mathbf{U}}_{1}^{H},
$$

where $\overline{\mathbf{V}}_{1}, \overline{\mathbf{V}}_{2}$ and $\overline{\mathbf{U}}_{1}$ denote the submatrices that contain the first $K$ columns of $\mathbf{V}_{1}, \mathbf{V}_{2}$ and $\mathbf{U}_{1}$, respectively. $\Sigma_{1}$ and $\Sigma_{2}$ are the diagonal matrices. 


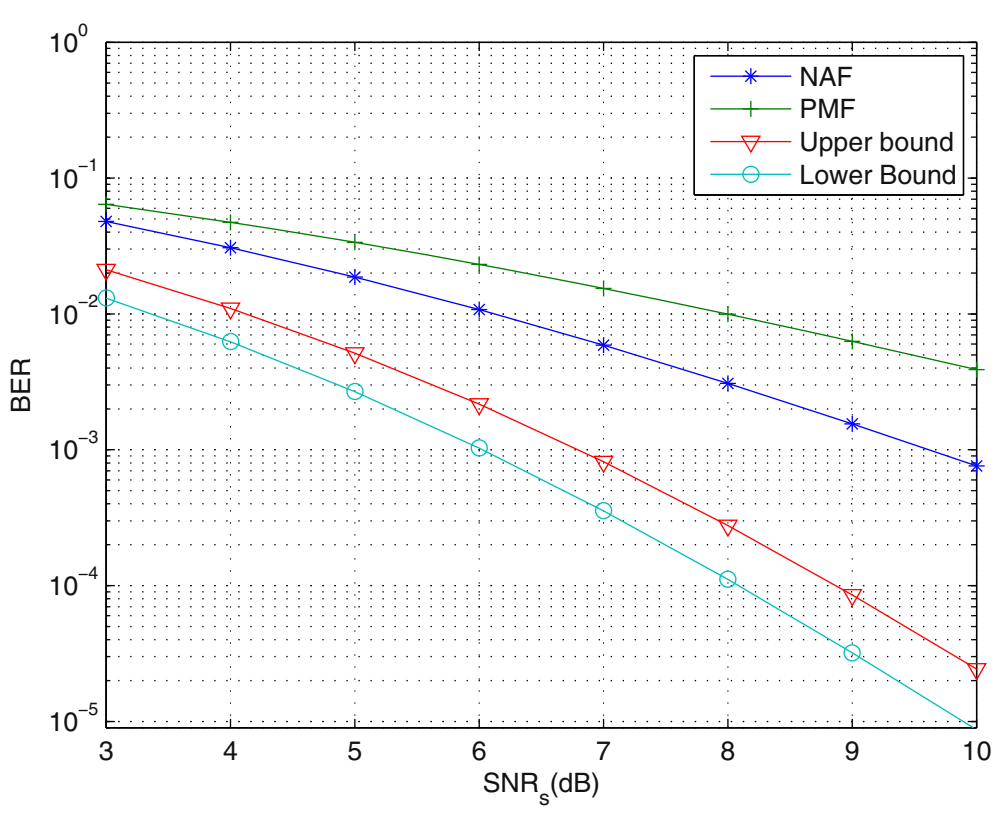

Fig. 3 BER vs SNR $. N_{s}=N_{r}=N_{d}=3$

Substituting (18)-(21) into (11), the MSE matrix can be calculated as follows

$$
\mathbf{M}\left(\Sigma_{1}, \Sigma_{2}\right)=\left(\mathbf{I}_{K}+\frac{\bar{\Lambda}_{1}^{2} \bar{\Lambda}_{2}^{2} \Sigma_{1}^{2} \Sigma_{2}^{2}}{\delta_{2}^{2} \mathbf{I}_{K}+\delta_{1}^{2} \bar{\Lambda}_{2}^{2} \Sigma_{2}^{2}}\right)^{-1}
$$

where $\bar{\Lambda}_{1}$ and $\bar{\Lambda}_{2}$ denote the diagonal matrices that contain the first $K$ columns of $\Lambda_{1}$ and $\Lambda_{2}$, respectively.
Therefore, the optimization problem of the AMSE can be rewritten as

$$
\min _{\sigma_{1, k}, \sigma_{2, k}} \sum_{k=1}^{K}\left(1+\frac{\lambda_{1, k}^{2} \lambda_{2, k}^{2} \sigma_{1, k}^{2} \sigma_{2, k}^{2}}{\delta_{2}^{2}+\delta_{1}^{2} \lambda_{2, k}^{2} \sigma_{2, k}^{2}}\right)^{-1},
$$

where $\sigma_{1, k}, \sigma_{2, k}, \lambda_{1, k}$ and $\lambda_{2, k}$ denote the $k$ th diagonal entry of $\Sigma_{1}, \Sigma_{2}, \Lambda_{1}$, and $\Lambda_{2}$, respectively, $\forall k \in\{1,2, \ldots, K\}$.

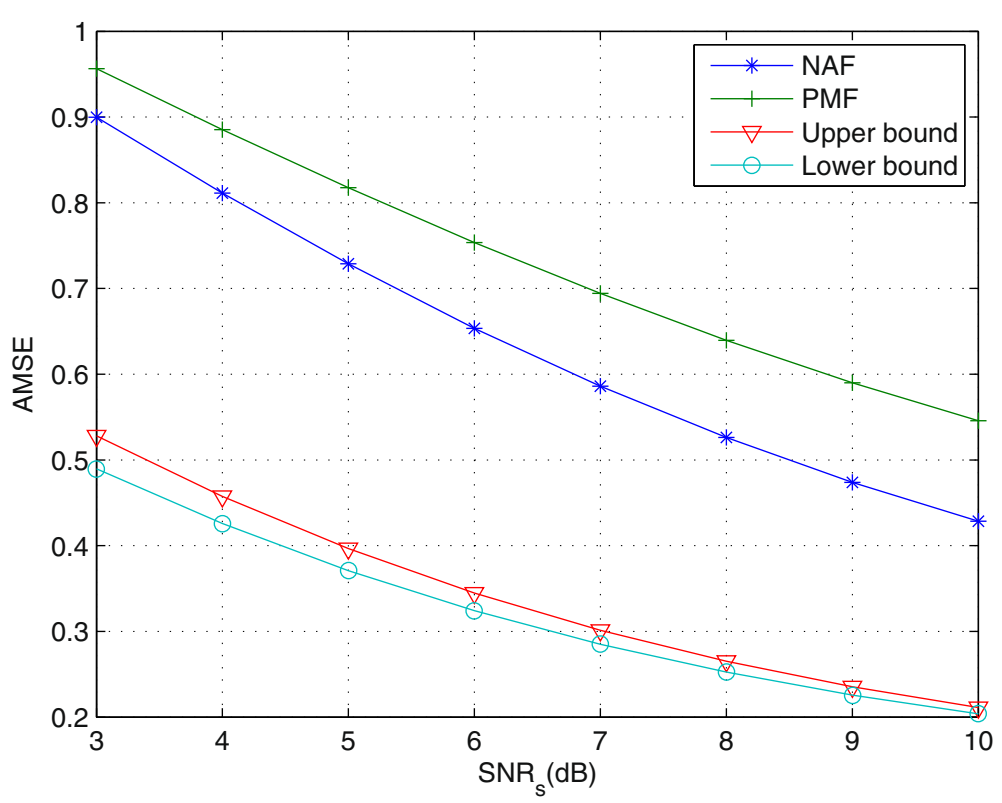

Fig. 4 AMSE vs SNR $. N_{s}=N_{d}=4, N_{r}=3$ 


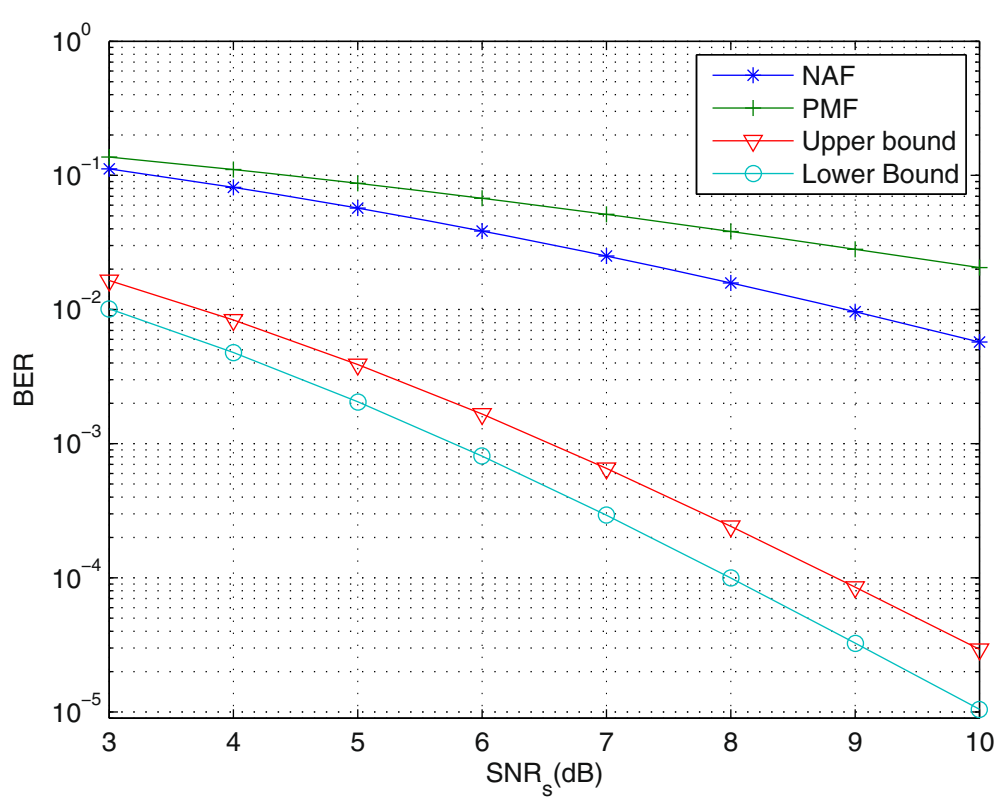

Fig. 5 BER vs $S N R_{s} . N_{S}=N_{d}=4, N_{r}=3$

The whole channel can be divided into $K$ subchannels with the joint precoding approach where each subchannel gain can be specified as $\lambda_{1, k}^{2} \lambda_{2, k}^{2}$, while $\Sigma_{1}$ and $\Sigma_{2}$ can be treated as the power allocation. It is obvious the power allocation is a key parameter for the optimization in the AF MIMO relay system. After substituting (20) and (21) into the power constraint (17), we obtain

$$
\sigma_{1, k}^{2}=a_{k},
$$

and

$$
\sigma_{2, k}^{2}=\frac{b_{k}}{\lambda_{1, k}^{2} a_{k}+\delta_{1}^{2}}
$$

where $a_{k}$ and $b_{k}$ are the power allocated to the $k$ th data stream at the source and the relay, respectively. Furthermore, taking $\bar{\lambda}_{1, k}^{2}=\lambda_{1, k}^{2} / \delta_{1}^{2}$ and $\bar{\lambda}_{2, k}^{2}=\lambda_{2, k}^{2} / \delta_{2}^{2}$ and replacing $\sigma_{1, k}, \sigma_{2, k}, \lambda_{1, k}$ and $\lambda_{2, k}$ in (23), the optimization problem can be expressed as follows

$$
\begin{aligned}
\min _{a_{k}, b_{k}} & \sum_{k=1}^{K}\left(\frac{\bar{\lambda}_{1, k}^{2} a_{k}+\bar{\lambda}_{2, k}^{2} b_{k}+1}{\bar{\lambda}_{1, k}^{2} a_{k}+\bar{\lambda}_{2, k}^{2} b_{k}+\bar{\lambda}_{1, k}^{2} \bar{\lambda}_{2, k}^{2} a_{k} b_{k}+1}\right), \\
\text { s.t. } & \sum_{k=1}^{K} a_{k} \leq P_{1}, \sum_{k=1}^{K} b_{k} \leq P_{2} .
\end{aligned}
$$

It is obvious that the abovementioned objective function is not convex [10]. Namely, it is difficult to get the optimal solution from (26). Although Rong et al. [12] has proposed an iterative algorithm for the optimal solution, the computational complexity is still very high. In order to reduce the computational complexity, an upper bound as a suboptimal solution was derived [14], where we can get the very close performance to an iterative algorithm. In the following, we will propose a lower bound to achieve the better performance but having a little high computational complexity comparing with the upper bound.

There are two known conventional bounds given by

$$
\frac{x+y+1}{x+y+x y+1} \leq \frac{x+y+2}{x+y+x y+1}
$$

and

$$
\frac{x+y+1}{x+y+x y+1} \geq \frac{x+y}{x+y+x y},
$$

where $x, y>0$ or $x<0, y<0, x y \neq 1$.

On the one hand, since $\bar{\lambda}_{1, k}^{2} a_{k}$ and $\bar{\lambda}_{2, k}^{2} b_{k}$ in (26) are positive values in our system, it is suitable to use the two bounds into the objective function. We substitute (27) into the objective function (26) and an upper bound can be calculated as

$$
\begin{aligned}
& \sum_{k=1}^{K}\left(\frac{\bar{\lambda}_{1, k}^{2} a_{k}+\bar{\lambda}_{2, k}^{2} b_{k}+2}{\bar{\lambda}_{1, k}^{2} \bar{\lambda}_{2, k}^{2} a_{k} b_{k}+\bar{\lambda}_{1, k}^{2} a_{k}+\bar{\lambda}_{2, k}^{2} b_{k}+1}\right) \\
= & \sum_{k=1}^{K}\left(\frac{1}{\bar{\lambda}_{1, k}^{2} a_{k}+1}+\frac{1}{\bar{\lambda}_{2, k}^{2} b_{k}+1}\right) .
\end{aligned}
$$

This optimization problem can be solved by two suboptimal solutions, i.e.,

$$
\min _{a_{k}} \sum_{k=1}^{K} \frac{1}{\bar{\lambda}_{1, k}^{2} a_{k}+1}, \quad \text { s.t. } \sum_{k=1}^{K} a_{k} \leq P_{1},
$$


and

$$
\min _{b_{k}} \sum_{k=1}^{K} \frac{1}{\bar{\lambda}_{2, k}^{2} b_{k}+1}, \quad \text { s.t. } \sum_{k=1}^{K} b_{k} \leq P_{2} .
$$

The abovementioned suboptimal solutions are developed by Rong with the MMSE criterion [14]. On the other hand, the lower bound can be similarly derived by using the inequality (28), where the lower bound can be denoted by $f(x, y)=(x+y) /(x+y+x y)$. It can be proved that this lower bound is a convex function, i.e.,

$$
\nabla^{2} f(x, y)=\frac{2}{(x+y+x y)^{3}}\left[\begin{array}{cc}
y^{2}(y+1) & -x y \\
-x y & x^{2}(x+1)
\end{array}\right] \succeq 0 .
$$

In the following, we derive another suboptimal solution which can be written as

$$
\begin{aligned}
\min _{a_{k}, b_{k}} & \sum_{k=1}^{K}\left(\frac{\bar{\lambda}_{1, k}^{2} a_{k}+\bar{\lambda}_{2, k}^{2} b_{k}}{\bar{\lambda}_{1, k}^{2} \bar{\lambda}_{2, k}^{2} a_{k} b_{k}+\bar{\lambda}_{1, k}^{2} a_{k}+\bar{\lambda}_{2, k}^{2} b_{k}}\right), \\
\text { s.t. } & \sum_{k=1}^{K} a_{k} \leq P_{1}, \sum_{k=1}^{K} b_{k} \leq P_{2} .
\end{aligned}
$$

Taking the Karush-Kuhn-Tucker (KKT) conditions [23], we get the solution of the optimization problem, which yields an equivalent function

$$
\begin{array}{r}
F=\sum_{k=1}^{K}\left(\frac{\bar{\lambda}_{1, k}^{2} a_{k}+\bar{\lambda}_{2, k}^{2} b_{k}}{\bar{\lambda}_{1, k}^{2} \bar{\lambda}_{2, k}^{2} a_{k} b_{k}+\bar{\lambda}_{1, k}^{2} a_{k}+\bar{\lambda}_{2, k}^{2} b_{k}}\right) \\
+v_{1}\left(\sum_{k=1}^{K} a_{k}-P_{1}\right)+v_{2}\left(\sum_{k=1}^{K} b_{k}-P_{2}\right),
\end{array}
$$

where $v_{1}$ and $v_{2}$ are the Lagrange multipliers. After making the tedious partial derivatives of equation (34), the solution of the unknown parameters $\left(a_{1}, a_{2}, \ldots, a_{k}\right)$ and $\left(b_{1}, b_{2}, \ldots, b_{k}\right)$ can be derived. Because of the partial derivatives in the calculation, the computational complexity of the lower bound is a little higher than that of the upper bound.

\section{Numerical results}

In this section, we analyze the derived lower bound for the AF MIMO relay system. The two-channel matrices are assumed to be distributed with $\mathcal{C N}(0,1)$. The SNRs at the relay and the destination are defined as $\mathrm{SNR}_{s}=P_{1} / \sigma_{1}^{2}$ and $\mathrm{SNR}_{d}=P_{2} / \sigma_{2}^{2}$, respectively.

We compare the upper bound of the proposed scheme with the initial amplify-and-forward (NAF) algorithm [12] or Pseudo match-and-forward (PMF) algorithm [24]. In the NAF-based scheme, the source precoding matrix is given by

$$
\mathbf{W}_{1}=\sqrt{\frac{P_{1}}{K}} \mathbf{I}_{K}
$$

and the relay precoding matrix is described as

$$
\mathbf{W}_{2}=\sqrt{\frac{P_{2}}{\operatorname{tr}(\Psi)}} \mathbf{I}_{N_{r}}
$$

where $\Psi=\mathbf{H}_{1} \mathbf{W}_{1}\left(\mathbf{H}_{1} \mathbf{W}_{1}\right)^{H}+\mathbf{I}_{N_{r}}$. In the PMF-based scheme, the matrix $\mathbf{W}_{1}$ is same as (30), while $\mathbf{W}_{2}$ is given by

$$
\mathbf{W}_{2}=\sqrt{\frac{P_{2}}{\operatorname{tr}\left(\left(\mathbf{H}_{1} \mathbf{H}_{2}\right)^{H} \Psi \mathbf{H}_{1} \mathbf{H}_{2}\right)}} \times\left(\mathbf{H}_{1} \mathbf{H}_{2}\right)^{H} .
$$

In order to compare with the PMF-based scheme, we take $N_{s}=N_{d}$ in the following analysis. Firstly, we consider a case of the same number of antennas at each node. Without loss of generality, we assume that $N_{s}=N_{r}=N_{d}=3$ and $K=2$. Figure 2 shows the AMSE of all algorithms for the fixed $\rho_{2}=10 \mathrm{~dB}$. The BER performance of the algorithms is demonstrated in Fig. 3. It is shown that the derived lower bound of the joint precoding scheme has a better performance than that of either NAF-based or PMF-based scheme. Comparing the lower bound with the upper bound, the difference of the AMSE is reduced as the SNR increases, which is shown in Fig. 2, and the two curves are almost overlapped at SNR around $10 \mathrm{~dB}$. However, the BER performance of the lower bound is slightly different from the upper bound, as shown in Fig. 3.

Subsequently, we consider another case of the different number of antennas. We take $N_{s}=N_{d}=4, N_{r}=3$ and $K=2$ in the simulations. The numerical results of the AMSE and the BER of the related algorithms are shown in Figs. 4 and 5, respectively. We also find that the lower bound is still superior than that of the previous schemes. The derived lower bound and upper bound approach each other, especially at the high SNR. This is consistent with the case of the same number of antennas. It implies that the derived lower bound is approaching to the true objective curve at the high SNR. In other words, the accuracy of the proposed lower bound is great guaranteed with the increment of the SNR.

\section{Conclusions}

We have presented a joint precoding scheme for the AF MIMO relay system. We derive a lower bound as the suboptimal solutions to overcome nonconvexity of the objective function. Numerical results show that compared with the previous schemes, the proposed scheme can obtain a great performance gain in terms of the SNR. In addition, the performance of the lower bound approaches to that of the existing upper bound, especially at the high SNR. Therefore, the accuracy of the proposed lower bound is guaranteed with the increment of the SNR. In our future work, we will extend this scheme to the case of imperfect CSI with the limited feedback, which is more practical in wireless relay networks. 


\section{Competing interests}

The authors declare that they have no competing interests.

\section{Acknowledgements}

This work was supported by MEST 2015R1A2A1A05000977, NRF, South Korea, National Nature Science Foundation of China (61201249, 61359153, 61272495), and the Brain Korea 21 PLUS Project, National Research Foundation of Korea.

\section{Author details}

${ }^{1}$ Department of Electronics and Information Engineering, Chonbuk National University, Baekje Road, Jeonju, South Korea. ${ }^{2}$ School of Information Science and Technology, Donghua University, Shanghai, China. ${ }^{3}$ School of Information Science and Engineering, Central South University, Lushangnan Road,

Changsha, China.

Received: 20 November 2014 Accepted: 7 May 2015

Published online: 19 June 2015

\section{References}

1. EC Van Der Meulen, Three-terminal communication channels. Adv. Appl. Prob. 3, 120-154 (1971)

2. TM Cover, AEL Gamal, Capacity theorems for the relay channel. IEEE Trans. Inform. Theory. 25(5), 572-584 (1979)

3. P Gupta, PR Kumar, The capacity of wireless networks. IEEE Trans. Inform. Theory. 46(2), 388-404 (2000)

4. JN Laneman, DNC Tse, GW Wornell, Cooperative diversity in wireless networks: Efficient protocols and outage behavior. IEEE Trans. Inform. Theory. 50(12), 3062-3080 (2004)

5. IE Telatar, Capacity of multi-antenna gaussian channels. European Trans. Telecom. 10(6), 585-595 (1999)

6. C Chae, T Tang, RW Heath, S Cho, MIMO relaying with linear processing for multiuser transmission in fixed relay networks. IEEE Trans. Signal Process. 56(2), 727-738 (2008)

7. Z Fang, Y Hua, JC Koshy, in Fourth IEEE Workshop on Sensor Array and Multichannel Processing. Joint source and relay optimization for a non-regenerative MIMO relay, (2006), pp. 239-243

8. X Tang, Y Hua, Optimal design of non-regenerative $\mathrm{MIMO}$ wireless relays. IEEE Trans. Wireless Commun. 6(4), 1398-1407 (2007)

9. O Muñoz-Medina, J Vidal, A Agustín, Linear transceiver design in nonregenerative relays with channel state information. IEEE Trans. Signal Process. 55(6), 2593-2604 (2007)

10. I Hammerstrom, A Wittneben, Power allocation schemes for amplify-and-forward MIMO-OFDM relay links. IEEE Trans. Wireless Commun. 6(8), 2798-2802 (2007)

11. W Guan, H Luo, Joint MMSE transceiver design in non-regenerative MIMO relay systems. IEEE Commun. Letters. 12(7), 517-519 (2008)

12. $Y$ Rong, $X$ Tang, $Y$ Hua, A unified framework for optimizing linear nonregenerative multicarrier MIMO relay communication systems. IEEE Trans. Signal Process. 57(12), 4837-4851 (2009)

13. Y Rong, in IEEE International Conference on Communications (ICC). Non-regenerative multicarrier $\mathrm{MIMO}$ relay communications based on minimization of mean-squared error, (2009), pp. 1-5

14. Rong, $Y$, Linear non-regenerative multicarrier MIMO relay communications based on MMSE criterion. IEEE Trans. Commun. 58(7), 1918-1923 (2010)

15. A Ikhlef, R Schober, Joint source-relay optimization for fixed receivers in multi-antenna multi-relay networks. IEEE Trans. Wireless Commun. 13(1), 62-74 (2014)

16. TX Tran, NH Tran, HR Bahrami, S Sastry, On achievable rate and ergodic capacity of NAF multi-relay networks with CSI. IEEE Trans. Commun. 62(5), 1490-1502 (2014)

17. Z Ding, T Wang, M Peng, W Wang, KK Leung, On the design of network coding for multiple two-way relaying channels. IEEE Trans. Wireless Commun. 10(6), 1820-1832 (2011)

18. Z Zhao, M Peng, Z Ding, W Wang, HH Chen, Denoise-and-forward network coding for two-way relay MIMO systems. IEEE Trans. Veh. Technol. 63(2), 775-788 (2014)

19. S Yadav, PK Upadhyay, S Prakriya, Performance evaluation and optimization for two-way relaying with multi-antenna sources. IEEE Trans. Veh. Technol. 63(6), 2982-2989 (2014)
20. L Sanguinetti, AA D'Amico, Y Rong, A tutorial on the optimization of amplify-and-forward MIMO relay systems. IEEE J. Selected Areas Commun. 30(8), 1331-1346 (2012)

21. DP Palomar, JM Cioffi, MA Lagunas, Joint Tx-Rx beamforming design for multicarrier MIMO channels: a unified framework for convex optimization. IEEE Trans. Signal Process. 51(9), 2381-2401 (2003)

22. JG Proakis, Digital Communications. (McGraw-Hill, New York, 1995)

23. S Boyd, L Vandenberghe, Convex Optimization. (Cambridge University Press, Cambridge, 2004)

24. PU Sripathi, JS Lehnert, in IEEE Conference Record of the Thirty-Eighth Asilomar Conference on Signals, Systems and Computers. A throughput scaling law for a class of wireless relay networks, vol. 2, (2004), pp. 1333-1337

\section{Submit your manuscript to a SpringerOpen ${ }^{\circ}$ journal and benefit from:}

- Convenient online submission

- Rigorous peer review

- Immediate publication on acceptance

- Open access: articles freely available online

- High visibility within the field

- Retaining the copyright to your article

Submit your next manuscript at $>$ springeropen.com 